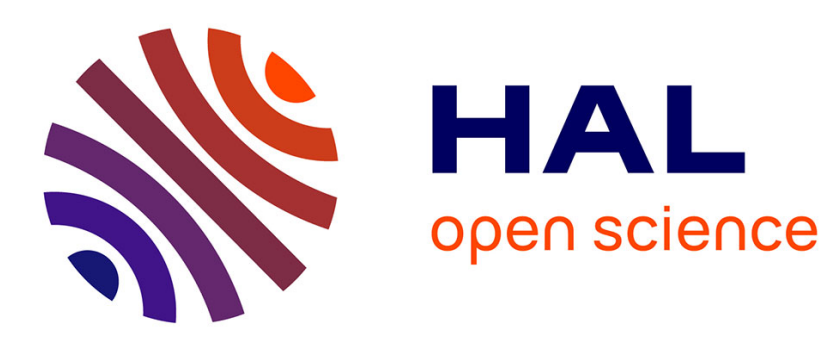

\title{
Thermal diffusivity determination by the photodeflection method. The influence of wave optical effects
}

\author{
H.-G. Walther, K. Muratikov, A. Glazov
}

\section{To cite this version:}

H.-G. Walther, K. Muratikov, A. Glazov. Thermal diffusivity determination by the photodeflection method. The influence of wave optical effects. Journal de Physique IV Proceedings, 1994, 04 (C7), pp.C7-291-C7-294. 10.1051/jp4:1994769 . jpa-00253298

\section{HAL Id: jpa-00253298 https://hal.science/jpa-00253298}

Submitted on 1 Jan 1994

HAL is a multi-disciplinary open access archive for the deposit and dissemination of scientific research documents, whether they are published or not. The documents may come from teaching and research institutions in France or abroad, or from public or private research centers.
L'archive ouverte pluridisciplinaire HAL, est destinée au dépôt et à la diffusion de documents scientifiques de niveau recherche, publiés ou non, émanant des établissements d'enseignement et de recherche français ou étrangers, des laboratoires publics ou privés. 


\title{
Thermal diffusivity determination by the photodeflection method. The influence of wave optical effects
}

\author{
H.-G. Walther, K.L. Muratikov* and A.L. Glazov \\ Friedrich-Schiller-University Jena, Institute for Optics and Quantum Electronics, Max-Wien Platz I, \\ 07743 Jena, Germany \\ * A.F. Ioffe Physicotechnical Institute of the Academy of Sciences of Russia, Politehnicheskaya 26, \\ 194021 St. Petersburg, Russia
}

\begin{abstract}
Possible approaches to thermal wave based measurement of thermal diffusivity are considered. The calculation of the transverse photodeflection signal in the framework of wave optics prove to be a more accurate approach compared to that of geometrical optics. The influence of the account of wave optical effects on the accuracy of the thermal diffusivity determination is theoretically analyzed under various conditions.
\end{abstract}

\section{INTRODUCTION}

A transverse photodeflection (TPD) technique has become of special interest within the last few years due to a possibility of determining the thermal diffusivity of solids [1-4]. This technique allows to carry out measurements locally, without contact and in a selected lateral (parallel to the sample surface) direction. In the case of thermally thick samples with large thermal diffusivity (compared with that of the surrounding deflection media) diffusivity can be measured directly [1]. Under other conditions, the thermal diffusivity determination is a more complicated procedure needing a numerical analysis of the TPD signal. The accuracy of the determination depends on the theoretical approach to the signal formation.

At present, the theory of TPD methods used for these purposes is based on the notions of geometric optics $[5,6]$. We showed elsewhere $[7,8]$ for a normal photodeflection signal and in ref. [10] for a transversal photodeflection signal that this approximation, neglecting the diffraction of a probe laser beam on the thermal lens (TL) and the probe beam intensity distribution in the region of the TL, can be responsible for a considerable difference between experimental and calculated data, especially for the phase of the TPD signal used for the thermal diffusivity determination.

\section{SIMULATION OF DIFFUSIVITY DETERMINATION}

In general, the thermal diffusivity measurement is based on a fit of the value from a dependence of the TPD signal on the lateral offset between pump and probe beams. To study theoretically the accuracy of the thermal diffusivity derived from geometrical optics compared to that of wave optics we have analyzed a fit procedure based on geometrical optics using simulated data from a wave optics model for samples with various thermal diffusivities and under various experimental conditions.

Principally, only two points of the dependence of the TPD signal phase on lateral offset $y_{0}$ can be used 
for the thermal diffusivity determination if all other experimental parameters are known. This approach is used in so called zero-crossing method [1], which we try to simulate also. For fitting we use two points of the phase shift dependence: $\varphi_{1}=\varphi\left(\mathrm{y}_{0} \rightarrow 0\right)$ and $\varphi_{2}=\Delta \varphi+\varphi\left(\mathrm{y}_{0} \rightarrow 0\right)$ at various $\Delta \varphi . \Delta \varphi=90^{\circ}$ corresponds to the zero-crossing method. First, we find the lateral offset $y_{0}$ at given $\Delta \varphi$ and the sample thermal diffusivity $\kappa_{0}$ using the signal in the wave optics approach

$$
\begin{gathered}
S(\omega)=\frac{2 I_{0} \eta}{\sqrt{\pi} \lambda r} \cdot \frac{d n}{d T} \int_{-\infty}^{\infty} d y \int_{-\infty}^{\infty} d k_{y} T\left(0, k_{y}, \omega\right) \exp \left(i k_{y} y-\gamma_{g} z_{0}+\frac{\left(\gamma_{g} r\right)^{2}}{4}-\frac{\left(y-y_{0}\right)^{2}}{r^{2}}\right) \\
\times i \operatorname{erf}\left(i \frac{y-y_{0}}{\sqrt{2} r}\right)\left[1+\operatorname{erf}\left(-\frac{\gamma_{g} r}{2}+\frac{z_{0}}{r}\right)\right],
\end{gathered}
$$

and then search the $\kappa_{\text {geom }}$ obeying the equation

$\Delta \varphi_{\text {geom }}\left(y_{0}, \kappa_{\text {geom }}\right) \equiv \varphi_{\text {geom }}\left(y_{0}\right)-\varphi_{\text {geom }}\left(y_{0} \rightarrow 0\right)=\Delta \varphi_{\text {geom }}$

using the geometrical optics expression for the signal

$$
S_{g}(\omega)=\frac{I_{0} \eta}{2 \sqrt{2 \pi} \lambda r} \cdot \frac{d n}{d T} \int_{-\infty}^{\infty} d k_{y} i k_{y} T\left(0, k_{y}, \omega\right) \exp \left(i k_{y} y_{0}-\gamma_{g} z_{0}+\frac{i \omega r^{2}}{4 \kappa_{g}}\right)\left[1+e r f\left(-\frac{\gamma_{g} r}{2}+\frac{z_{0}}{r}\right)\right] \text {, }
$$

vwhere $\mathrm{dn} / \mathrm{dT}$ is the derivative of the medium refractive index; $\lambda$ is the wavelength of the probe beam; $\mathrm{T}\left(\mathrm{k}_{\mathrm{x}}, \mathrm{k}_{\mathrm{y}}, \omega\right)$ is the Fourier transform of the non-stationary component of the object surface temperature $\mathrm{T}(\mathrm{x}, \mathrm{y}, \mathrm{z}=0, \mathrm{t})$ over the coordinates $\mathrm{x}$ and $\mathrm{y}$ and time; $\gamma_{\mathrm{g}}=\left(\mathrm{k}_{\mathrm{y}}+\mathrm{i} \omega / \kappa_{\mathrm{g}}\right)^{1 / 2} ; \kappa_{\mathrm{g}}$ is the thermal diffusivity of the medium near the object; $r$ is the probe beam radius.

Note that the signal amplitude equals zero at $\mathrm{y}_{0}=0$, hence determining $\varphi(0)$ exactly is impossible experimentally. In general, we could use another point or points for fitting. On the other hand, $\varphi \rightarrow$ const at $y_{0} \rightarrow 0$, and we can use the limit $\varphi(0)$ in simulations as a reference point.

Calculations show that the dependence of the lateral offset on the thermal diffusivity is not monotonic. Hence, problems of multi-valued solutions can arise in the diffusivity determination if few experimental data is used. This actually happens at small $\Delta \varphi$ and/or $\kappa$.

The "fitting" of the thermal diffusivity at $\Delta \varphi=90^{\circ}$ is shown in Fig.1. The cross of dashed straight lines corresponds to the wave optics solution of Eq. 1 for all diffusivities. Other curves represent dependencies $\Delta \varphi_{\text {geom }}$ on the normalized thermal diffusivity for four materials with various $\kappa_{0}$ and for the signal calculated using geometrical approach at corresponding $y_{0}$. The crossing of the horizontal line by these curves gives a "fitted"

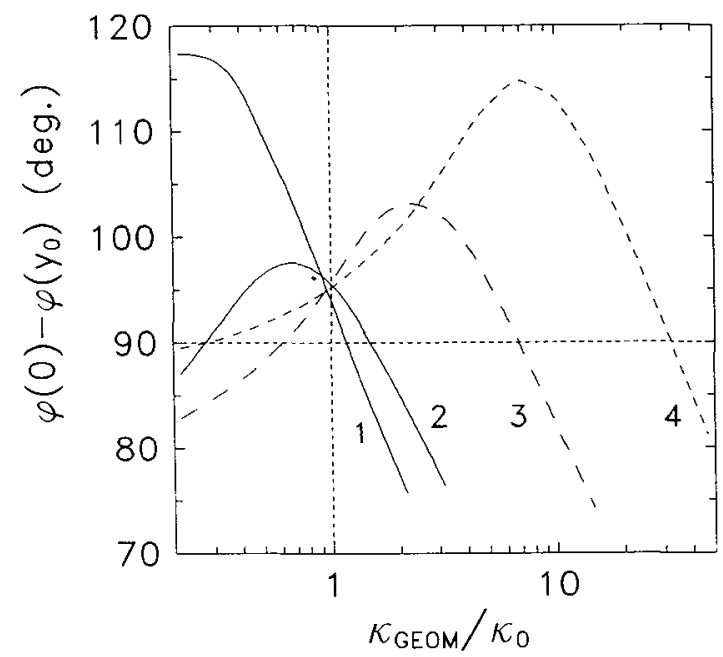

Fig. 1. The "fitting" of diffusivity. $1 . \kappa_{0}=0.3 \mathrm{~cm}^{2} / \mathrm{s}$. 2. $\kappa_{0}=0.1 \mathrm{~cm}^{2} / \mathrm{s} .3 . \kappa_{0}=0.03 \mathrm{~cm}^{2} / \mathrm{s} .4 . \kappa_{0}=0.01 \mathrm{~cm}^{2} / \mathrm{s}$. value of $\kappa_{\text {geom }}$. These dependencies have a maximum greater than $90^{\circ}$ and two solutions, one smaller and 
one greater than initial $\kappa$.

Fig. 2 shows the two branches of solutions vs $\kappa_{0}$. The greater solution decreases monotonically with the increase of $\kappa_{0}$ and about the original $\kappa_{0}$ if $\kappa_{0}$ is greater than the thermal diffusivity of deflecting medium $\left(0.2 \mathrm{~cm}^{2} / \mathrm{s}\right.$ in the case of air). The dashed curves represent similar dependencies with a simple geometrical optics approach with $r=0$ being used for the fitting. One can see that accounting for the finite probe beam radius within the framework of geometrical optics slightly improves the fitting accuracy. One can see from figure 2 that the neglect of the wave optics effects can lead to an error of two orders of magnitude or more.

We have not presented here the results of the simulation at high modulation frequencies, because the wave optics effect (see for instance in ref.[7] for normal photodeflection signal) and the error will be greater.

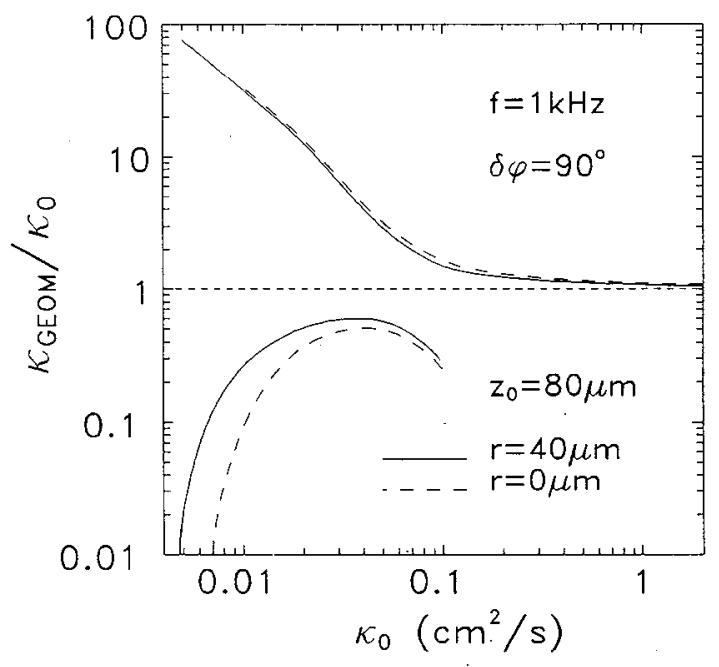

Fig.2.The error of the diffusivity determination by geometrical optics approach for simulated data.

\section{EXPERIMENTAL DETECTION OF DIFFUSIVITY}

For an experimental study of the influence of wave optics effects on the thermal diffusivity determination, some metal samples were measured.

$\mathrm{An} \mathrm{Ar}^{+}$-ion laser was used for thermal wave generation. The radius of the heating beam was as small as $7 \mu \mathrm{m}$ to reduce its influence in fitting. A monoblock by Phototherm Dr.Petry GmbH was used as a detection system. The radius of the probe beam was $45 \mu \mathrm{m}$. The vertical offset was estimated by crossing the probe beam center by a sample edge and chosen about $100 \mu \mathrm{m}$. A position of the probe beam and the sample was fixed, and we scanned the heating beam across the probe beam direction.

Experimental investigations were made for tungsten and argentan with air as a deflecting medium. The thermal diffusivity was determined using a three parameter least-squares-fitting of the TPD signal phase vs lateral offset for the thermal diffusivity of the sample, that of air and the vertical offset. Table 1 containes a summary of the results of the fit at two modulation frequencies. $\sigma$ is the phase variance,

$$
\sigma=\sqrt{\sum \frac{\left(\varphi_{\text {exper }}-\varphi_{\text {theor }}\right)^{2}}{N}} .
$$

Literature gives the following data for the thermal diffusivities: $\kappa_{0}$ is $0.68 \mathrm{~cm}^{2} / \mathrm{s}$ at $300^{\circ} \mathrm{K}$ and $0.61 \mathrm{~cm}^{2} / \mathrm{s}$ at $400^{\circ} \mathrm{K}$ for tungsten [9], and $\kappa_{0}$ is $0.076-0.09 \mathrm{~cm}^{2} / \mathrm{s}$ at $300^{\circ} \mathrm{K}$ for argentan [10].

So, one can see that at the frequency of $1 \mathrm{kHz}$ the both approaches give similar results which is in a correspondence with literature data. A diffusivity of $0.28 \mathrm{~cm}^{2} / \mathrm{s}$ correspondes to the thermal diffusivity of air at $360^{\circ} \mathrm{K}$ under normal conditions. The increase of the modulation frequency results in a difference between all parameters fitted within the frameworks of the two approaches. In the case of argentan, the geometrical one gives no reasonable value of the diffusivity at $5 \mathrm{kHz}$. 
Table 1. Results of the fit procedure for tungsten and argentan.

\begin{tabular}{|l|cc|cc|cc|cc|}
\hline sample & \multicolumn{4}{|c|}{ tungsten } & \multicolumn{3}{c|}{ argentan } \\
\hline $\mathrm{f}(\mathrm{kHz})$ & \multicolumn{2}{|c|}{1.0} & \multicolumn{2}{c|}{5.0} & \multicolumn{2}{c|}{1.0} & \multicolumn{2}{c|}{5.0} \\
\hline approach & wave & geom. & wave & geom. & wave & geom. & wave & geom. \\
\hline$\kappa_{0}\left(\mathrm{~cm}^{2} / \mathrm{s}\right)$ & 0.64 & 0.63 & 0.69 & 0.75 & 0.069 & 0.069 & 0.05 & 0.006 \\
$\kappa_{\text {air }}\left(\mathrm{cm}^{2} / \mathrm{s}\right)$ & 0.28 & 0.28 & 0.23 & 0.30 & $\begin{array}{l}0.28 \\
102\end{array}$ & 0.29 & 0.22 & 0.31 \\
$\mathrm{z}_{0}(\mu \mathrm{m})$ & 102 & 102 & 101 & 104 & 98 & 101 \\
$\sigma(\mathrm{deg})$. & 2.7 & 2.5 & 1.6 & 3.4 & 3.5 & 3.2 & 4.2 & 5.5 \\
\hline
\end{tabular}

\section{CONCLUSIONS}

The use of the conventional approach for the interpretation of the transverse photo-deflection signal in the framework of geometrical optics often leads to errors in the determination of the thermal diffusivity. A wave optics approach to the formation of the transverse photodeflection signal has to be used in the following cases.

1. The thermal diffusivity to be measured is small compared to that of the deflecting medium.

2. The measurements need a high frequency, for instance, to determine the thermal diffusivity of thin samples or films or to make measurements with a high lateral resolution.

3. The measurements need a large probe beam radius, for instance, to determine the thermal diffusivity of large samples.

4. The phase shift used in fitting is small.

\section{ACKNOWLEDGEMENTS}

The support of this research by the Alexander von Humboldt Foundation of Germany is gratefully acknowledged. The authors would also like to thank Phototherm Dr.Petry GmbH for the the opportunity to use "mirage-effect" monoblock for the measurements.

\section{References}

[1] Kuo P.K., Sendler E.D., Favro L.D., Thomas R.L., Kim D.S., Zhang Shu-yi, Inglehart L.J., Fournier D., Boccara A.C., and Yacouby N., Can.J.Phys. 64 (1986) 1165-1167.

[2] Salazar A., Sanchez-Lavega A., and Fernandez J., J.Appl.Phys. 69 (1991) 1216-1223.

[3] Figari A., J.Appl.Phys. 71 (1992) 3138-3142.

[4] Rantala J., Jaarinen J., and Kuo P.K., Appl.Phys A 55 (1992) 586-695.

[5] Jackson W.B., Amer N.M., Boccara A.C., and Fournier D., Appl. Optics 20 (1981) 1333-1344.

[6] Aamodt L.C., and Murphy J.C., J.Appl.Phys. 52 (1981) 4903-4914.

[7] Glazov A., and Muratikov K., Opt. comm. 84 (1991) 283-289.

[8] Glazov A., and Muratikov K., Photoacoustic and Photothermal Phenomena III, Springer Series in Optical Sciences 69, (Springer, Berlin, 1991) pp. 528-530.

[9] Lide D.P.(ed.) Handbook of Chemistry and Physics (CRC Press, 1994).

[10]Lax E.(ed.) Taschenbuch für Chemiker and Physiker 1, (Springer, Berlin, 1967). 